\title{
Effect of the Fixation Patterns on Magnetic Characteristics of Amorphous Glass-Coated Sensoric Microwires
}

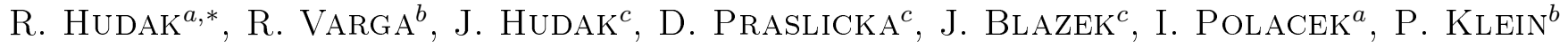 \\ ${ }^{a}$ Department of Biomedical Engineering and Measurement, Faculty of Mechanical Engineering, \\ Technical University of Kosice, Letna 9, 04200 Kosice, Slovakia \\ ${ }^{b}$ Department of Condensed Matters Physics, Institute of Physics, Faculty of Science, P. J. Safarik University, \\ Park Angelinum 9, 04154 Kosice, Slovakia \\ ${ }^{c}$ Department of Aviation Technical Studies, Faculty of Aeronautics, Technical University of Kosice, \\ Watsonova 47, 04001 Kosice, Slovakia
}

\begin{abstract}
Some of the frequent reasons of titanium implant failures in human body are incorrect biomechanical interactions within the tissue-implant interface and inflammatory processes arising around the implant's application area. For both processes, it is crucial to locate them and intervene in time. One of the monitoring possibilities of the mentioned processes is the application of amorphous glass-coated sensoric microwires (AGCSM). Magnetic characteristics of these microwires are influenced by both mechanical tension (magnetoelastic interaction of the magnetic moment with mechanical stress) and by temperature (different coefficient of thermal expansion of the metal core and glass coating). The key task, in order to change the magnetic characteristics of AGCSM from both clinical and scanning point of view, appears to be the fixation pattern of AGCSM in the implant's body. The presented study shows the impact of four types of AGCSM fixation patterns (at one ending, at both endings, in the middle and along the entire length) on the thermal response process tested in laboratory conditions. The obtained results will enable to establish the AGCSM fixation methodology in the implant's body in order to achieve optimal output temperature parameters form the implant and the tissue-implant interface by magnetic contactless measurements.
\end{abstract}

DOI: $10.12693 /$ APhysPolA.126.417

PACS: 87.85.G-, 87.85.fk, 85.70.Ay

\section{Introduction}

The application of micro-sensors in implants for monitoring of biomechanical parameters or other quantities is not new. Several studies have already been published describing similar applications. They include, for example, the MicroStrain system [1], systems using the low-frequency RFID technology [2] or outputs by the Charité institute (Julius Wolff Institute for Biomechanics and Musculoskeletal Regeneration (Universitatsmedizin Berlin, Germany) [3, 4]. The advantage of these systems lies in their dimensions and non-invasiveness; however currently the requirements of biocompatibility, price but also constant miniaturisation are increasing. One of the possibilities is the application of amorphous glasscoated sensing microwires (AGCSM). These microwires are composite materials consisting of a metallic nucleus $(d=1-20 \mu \mathrm{m})$ and an insulating glass coating $(d=2-$ $20 \mu \mathrm{m})$ [5]. Magnetic characteristics of these microwires are influenced by both mechanical tension (magnetoelastic interaction of the magnetic moment with mechanical stress) and by temperature (different coefficients of thermal expansion of the metal core and glass coating). The presented study shows the impact of four types of AGCSM fixation in the implant body (at one ending, at

*corresponding author; e-mail: radovan.hudak@tuke.sk both endings, in the middle and along the entire length) on the thermal response process tested in laboratory conditions.

\section{Materials and methods}

The research of the effect of fixation on magnetic characteristics of AGCSM and thus also on the quantity being sensed, which was temperature, consisted of the following steps: AGCSM manufacturing (determining the chemical composition of the microwire's metal core, producing a pre-alloy with the required chemical composition, manufacturing the microwire, testing magnetic bistability, annealing the microwire, measuring the critical fields of the domain wall and evaluation), manufacturing of test implants, fixation of microfibers and producing outputs (diagrams of the dependency of the critical field on temperature with individual types of fixation).

To simulate fixation of the microwire in the implant we used plates having dimensions of $50 \times 4 \times 2 \mathrm{~mm}$, which had a slot with dimensions $25 \times 1 \times 0.1 \mathrm{~mm}$, representing a cavity in the implant. These plates were manufactured by one of the technologies of additive manufacturing (AM), specifically by the technology of direct metal laser sintering (DMLS) using the device EOSINT M280 (EOS, Germany) from the alloy of titanium Ti-6Al-4V (Grade 5).

The position of the microfiber in the test plates was verified using the system of Metrotom 1500 (Carl Zeiss, 
Germany), which operates on the principle of industrial CT.

The measurements ascertained the effect of the method of fixation of the $\mathrm{Fe}_{78} \mathrm{~W}_{5} \mathrm{~B}_{17}$ microwire (25 mm long, with Curie temperature $T_{C}=180^{\circ} \mathrm{C}$ ) on the plate, simulating an implant, on the critical field $H_{S W}$ and, at the same time, its temperature dependency. The measurements were performed within the temperature range of $30^{\circ} \mathrm{C}$ to $170^{\circ} \mathrm{C}$ with a step of $10^{\circ} \mathrm{C}$. The microwire was fixed to the implant in four different ways: on one end (Fig. 1a), on two ends (Fig. 1b), in the middle (Fig. 1c) and along the entire length (Fig. 1d).
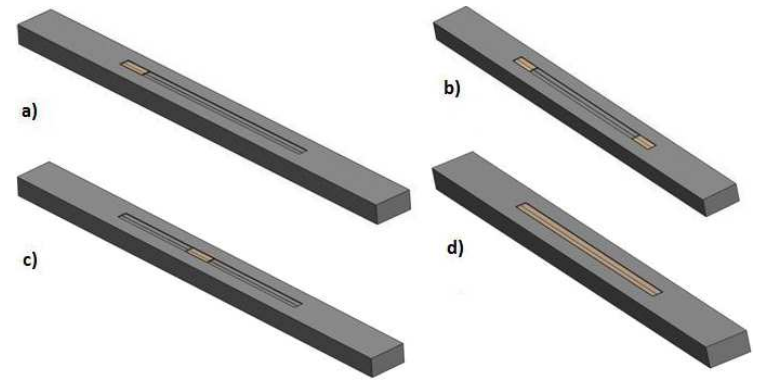

Fig. 1. Different method of AGCSM fixation on the Ti alloy a) single end, b) both ends, c) center, d) whole wire.

We used the measurements to produce diagrams of the dependencies of the critical field on the temperature with individual types of fixation, which monitored the sharpest drop of the critical field in a narrow temperature range. This sharp change of temperature, that enabled us to measure temperature in a sensitive way, appears to be below the Curie temperature. Therefore, it is necessary for the microwire to have the Curie temperature on the level of approximately $50^{\circ} \mathrm{C}$. For comparison we used the dependency of the critical field on the temperature of the microwire without fixation, where the sharpest change of the critical field at the frequency of $1000 \mathrm{~Hz}$ is between 120 and $140^{\circ} \mathrm{C}$.

\section{Results}

For biomedical applications it is necessary to monitor the sharpest drop in temperature within the appropriate temperature range: $35-42{ }^{\circ} \mathrm{C}$. Figure 2 shows that the sharpest drop in the required temperature range is at $100 \mathrm{~Hz}$ and with fixation along the entire length.

With a non-fixed loose microwire the sharpest drop was at $130^{\circ} \mathrm{C}$; with fixation along the entire length this change fell to $50^{\circ} \mathrm{C}$, which is a shift of $80^{\circ} \mathrm{C}$ only as a result of application of external pressure forces caused by solidification of the fixation medium. This solidification contributes to the magnetoelastic effect.

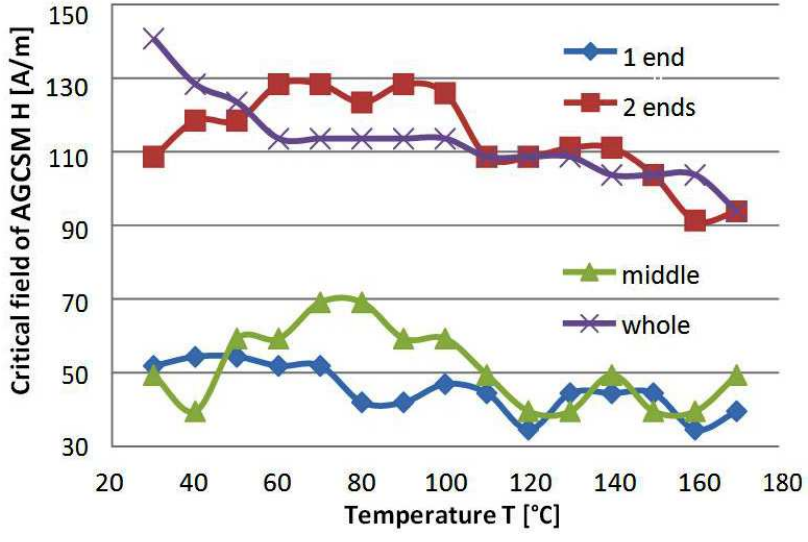

Fig. 2. Temperature dependence of the switching field measured for different methods of fixation.

\section{Conclusions}

The experimental measurements showed that the best way to fix the microwire is along the entire length (in case of the tested type of the microwire $\mathrm{Fe}_{78} \mathrm{~W}_{5} \mathrm{~B}_{17}$ ). However further studies will be needed to deal with quantification of the applied fixation material and its effect on the measured critical fields and thus also the temperature, analysis, selection and testing of an optimal biocompatible fixation material and testing of the effect of the microwire position on the measured data. An equally important area will be the testing of the sensing system with analysis of the range and thus the depth of human body scanning.

\section{Acknowledgments}

Presented manuscript was supported by project Research of New Diagnostic Methods in Invasive Implantology, MŠSR-3625/2010-11, Stimuls for Research and Development of Ministry of Education, Science, Research and Sport of the Slovak Republic.

\section{References}

[1] S.W. Arms, C.P. Townsend, Proc. NSF-ESF Joint Conf. Struct. Health Monitor., Strasbourg, France, October 3-5, 2003.

[2] Ch. Moss, W. Sass, N. Weinrich, K. Seide, J. Müller, Proc. SOMSED'09 1, 97 (2009).

[3] P. Damm, V. Schwachmeyer, J. Dymke, A. Bender, G. Bergmann, Cnlin. Biomech. 28, 530 (2013).

[4] G. Bergmann, F. Graichen, A. Rohlmann, A. Bender, B. Heinlein, G. Duda, M.O. Heller, M.M. Morlock, Bio-Med. Mater. Eng. 20, 65 (2010).

[5] R. Varga, T. Ryba, K. Saksl, V. Zhukova, A.P. Zhukov, Key Eng. Mat. 495, 236 (2011). 